\title{
Effects of Taping and Elastic Bandage on Postural Control in Athletes with Patellofemoral Pain: A Randomized Control Trial
}

\author{
Seyed Mojtaba Ojaghi ${ }^{1}$, Fahimeh Kamali ${ }^{2}$, Ali Ghanbari ${ }^{2}$, Samaneh Ebrahimi ${ }^{1}$, Ahmad Reza Nematollahi $^{1}$
}

'Student Research Committee, Shiraz University of Medical Sciences, Shiraz, Iran.

${ }^{2}$ School of Rehabilitation, Shiraz University of Medical Sciences, Shiraz, Iran.

\begin{abstract}
Background: Patellofemoral pain syndrome (PFPS) is the most common overuse syndrome in athletes. The aim of this study was to compare the effects of taping and elastic bandage on postural control in athletes with PFPS. Materials and Methods: Fifteen males and 19 females with PFPS participated in this clinical trial study for more than 1 month and were randomly divided into two groups; group 1 was taped based on McConnell method and in group 2 elastic bandage was used. The static postural control in both groups was measured before and after interventions using the force- plate through measuring the center of pressure (COP) and estimation of differences between center of pressure and center of mass (COP-COM moment arms) in AP and ML directions. Moreover, dynamic postural control was measured by star excursion balance test (SEBT). Paired t-test and covariance analysis were used for analysis of the data. Results: Results indicated that after taping reach distances increased significantly $(\mathrm{p}<0.05)$ in anterior, anterolateral, lateral and posterior directions but after elastic bandage reach distances increased in posterior, posteromedial and medial directions. After both taping and bandage, COP and COP-COM moment arm measures did not show significant differences. Conclusion: The findings of this investigation showed that in athletes with patellofemoral pain, taping and elastic bandage improved dynamic postural control. However, dynamic methods are successfully used to assess the effects of taping and bandage on postural control. Static variables compared with dynamic measures potentially lack the ability to detect subtle differences of postural control in athletes with PFPS. [GMJ. 2015;4(3):82-89]
\end{abstract}

Keywords: Postural Control; Taping; Bandage; Patellofemoral Pain Syndrome

\section{Introduction}

$\mathrm{P}$ atellofemoral pain is a common injury among young athletes. Up to $40 \%$ of clinical visits for knee problems are attributed to patellofemoral pain [1]. Lateral tracking of the patella at femoral trochlear groove has an important role in the development of pa-

\section{GMJ}

2013 Galen Medical Journal

Fax: +98 7312227091

PO Box 7461686688

Email:info@gmj.ir tellofemoral pain [2].

Based on previous research, while in healthy people in whom the vastus medialis oblique and vastus lateralis muscles concurrently contract, in patients with patellofemoral pain the vastus medialis oblique muscle contraction has a delayed onset related to vastus lateralis muscle contraction [3]. Quadriceps neu-

\footnotetext{
Correspondence to:

Fahimeh Kamali, Associate Professor, PT, School of Rehabilitation, Shiraz University of Medical Sciences, Shiraz, Iran.

Telephone Number: (+98) 71-36271551

Email Address :Fahimehkamali@hotmail.com
} 
romuscular deteriorations could be a result of the proprioception deficits due to patellar maltracking and the surrounding soft tissues painful irritation.

Physiotherapists and athletic trainers commonly use taping and other orthoses for treatment of the athletes who suffer from patellofemoral pain [4]. McConnell taping corrects patellar mal-positioning, enhances vastus medialis activity and finally reduces pain $[5,6]$. Also, elastic bandages could be used for various musculoskeletal injuries such as knee osteoarthritis (OA). Elastic bandage affects knee proprioception and postural control in patients with knee OA. Using an elastic bandage around the knee increases the cutaneous contribution in joint position sense. Proprioception initiates reflexes that help stabilize the limb and protect it from excessive movements via proprioceptive stretch reflex [7]. This study hypothesized that both taping and standard elastic bandage could cause functional improvements through proprioception enhancement.

Previous studies have shown that measurement of postural control is important in establishing the level of neuromuscular performance following an injury [8]. Besides, assessment of neuromuscular function through reliable methods enable clinicians to understand the impact of injury and influence of interventions on this system [9]. Therefore, current study assessed the effects of taping and bandage on static and dynamic postural control. Force-plate is sensitive to the center of pressure (COP) changes or sway area; therefore, by measuring the COP variable during one leg stance, we could assess the static postural control.

One type of postural control test which is more functional to assess athletes is star excursion balance test (SEBT). Previous studies have reported that SEBT has enough sensitivity to indicate dynamic postural control deficits in patients with Patellofemoral pain syndrome (PFPS), chronic ankle instability (CAI) and ACL-deficient limb [10].

To the best of our knowledge, no study has compared the effects of taping and standard elastic bandage on postural control in athletes with PFPS through dynamic and static meth- ods concurrently. Hence, the main objectives of this study are to firstly investigate the effects of McConnell taping and elastic bandage on static and dynamic postural control and secondly to compare the ability of these methods to detect differences of postural control after intervention among athletes with PFPS. This study hypothesized that: 1) taping and elastic bandage application in athletes with PFPS improve postural control ability, 2) postural control assessment by SEBT and COPCOM moment arm measures has a more potential to detect postural control differences after intervention.

\section{Materials and Methods}

\section{Subjects}

Thirty four participants (15males aged $25+/$ 2.06 ), height $174.6+/-4.8 \mathrm{~cm}$, weight $68+/-$ $9.6 \mathrm{~kg}$ and 19 females aged $23+/-1.91$, height $162.5+/-7.18 \mathrm{~cm}$, weight $56.92+/-4.24 \mathrm{~kg}$ all of whom had anterior knee pains volunteered to participate in this study. Sample size with $95 \%$ confidence interval for $\alpha=0.05$ was calculated from previous research [11]. The participants who had a history of regular sport activity at least 6 hours a week, had anterior knee pain or retro-patellar pain at least for 1 month, suffered from pain during step-up and step-down or squat, tenderness at facet joints, those in whom step-down eccentric test was positive were included in this study. Exclusion criteria consisted of symptoms initiating less than 1 month ago, symptoms related to direct trauma, ligamentous laxity and/or other injuries such as ACL injury at tested leg and surgery at the tested knee during past 3 months and pain due to systemic disorders.

\section{Ethical Statement}

All subjects signed an informed consent form and the study was approved by Shiraz University of Medical Sciences Research and Ethics Committee (code: CT-90-5787). In addition, this study was registered in Iranian Registry of Clinical Trials (IRCT) (RCT code: IRCT201112268524N1).

\section{Protocols}

Participants were divided into two groups; 
group 1 was taped based on McConnell method and group 2 was wrapped with elastic bandage. Participants were randomly distributed in each group based on paired and single numbers. Postural control capability in both groups was measured before and after interventions (taping or standard elastic bandage) by force-plate and SEBT.

\section{Assessment of Static Postural Control}

For participants, three sessions of 20 seconds of single-leg stance were performed at their symptomatic side on a force-plate. During static test, the opposite leg was held in 30 degrees knee flexion without weight bearing. This procedure was performed by force-plate (type 9286AA, Kistler Switzerland). Raw data from force-plate were collected in sampling rate of $100 \mathrm{~Hz}$ and then low pass was filtered with a cut-off frequency of $5 \mathrm{~Hz}$. Then, three trials average were used for final analysis. COP displacement and velocity measures at the anterior- posterior and medial- lateral directions before and after taping or elastic bandage were used to assess the effects of interventions on static postural control.

\section{Calculation of Center of Pressure}

In this study, four traditional COP-based dependent variables were calculated. Mean COP displacement and mean velocity in both anteroposterior and mediolateral directions were calculated.

\section{Estimation of COP-COM Moment Arms}

Estimates for COM displacement were found by following formula $\lceil 12\rceil$ :

$$
\Delta \operatorname{COM}_{\mathrm{x}}=\int_{0}^{t} \int_{0}^{t}(f x / M) d t
$$

Then, COP-COM moment arms in AP and ML directions were calculated.

\section{Assessment of Dynamic Postural Control}

Star excursion balance test was used for assessment of dynamic postural control [13]. Participants stood bare foot in the middle of a grid on the leg to be tested. The grid was laid on the floor with eight lines extending out at $45^{\circ}$ from the center of the gird. Foot position was controlled by aligning the heel with the center of the grid and great toe with the anteriorly projected line. Before testing, subjects were instructed to hold their hands on the pelvis. Participants were asked to maintain single-leg stance while reaching the opposite leg as far as possible along each of the eight lines and then returned to bilateral stance at the start position. Each participant performed this trial three times for each of the lines (Figure1). The measures were normalized by participant's leg length values (ASIS to the distal tip of the medial malleolus in supine position) and finally the mean of scores was recorded. Each trial was repeated if the examiner felt subjects used any considerable support from the reaching leg while they touched down. Before testing, in each direction, participants performed each maneuver four times and then had 5 minutes rest prior to performing the test. Each of directions was named according to the direction of movement around the gird in relation to the standing leg.

\section{Application of Patellar Taping}

After assessment of static and dynamic postural controls, all patients were directed to lie supine with knee-extended position. The first layer was applied from lateral femoral condyle to the posterior and medial femoral condyle. The second layer was dressed from the lateral border of the patellar bone to medial

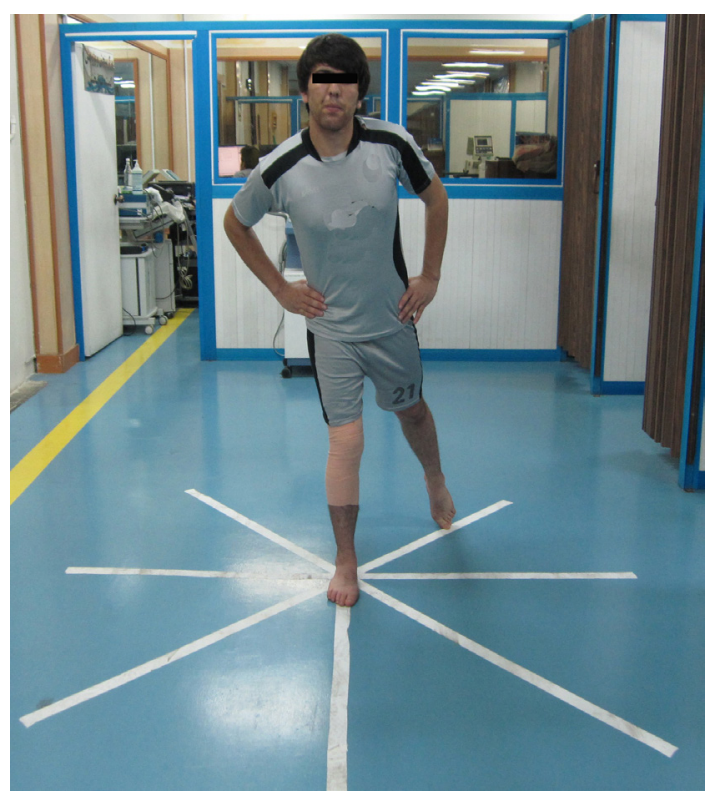

Figure1. Picture shows posteromedial reach direction of SEBT while standard elastic bandage application. 
condyle of the femur. This way, we gathered the soft tissue over the medial condyle. The last layer was attached from the middle of the patella to the medial femoral condyle. In this study, the patellar taping method was used because of some reports describing that the patellar position changed significantly following the application of McConnell taping [14]. The criterion for successful taping was pain reduction on VAS after applying the tape. If it did not happen, the procedure was repeated. After application of taping on the symptomatic side, the participants were allowed to have usual physical activity such as walking for 15 minutes. Then, static and dynamic postural control tests were performed by force-plate and SEBT.

\section{Application of Standard Elastic Bandage}

On taping and after assessment of static and dynamic postural control, the participants were directed to lie supine; we also performed wrapping of the knee with elastic bandage. The characteristics of bandage size were as follows: width: $10 \mathrm{~cm}$, length: $5.5 \mathrm{~m}$, product of Cramer ${ }^{\circledR}$ company (made in U.S.A). Standard elastic bandage was applied as recommended [7] so that the applied force did not overpressure the patellofemoral joint. The main objective was the acquisition of supportive effect from standard elastic bandage. Bandage covered the area from mid-thigh to mid-calf without a window for the patella, and thus this method allowed us to replicate the bandage easily (Figure1). Accordingly, pain intensity was examined by VAS. After applying the bandage on the symptomatic knee, similar to the taping group of participants, this group also did usual activities such as walking, step-up and down for 15 minutes. Then, static and dynamic postural control tests were performed.

\section{Statistical Analysis}

KS test demonstrated that all data were distributed normally. Paired t-test was used to assess the effects of taping and bandage on all static and dynamic postural control measures. In addition, analysis of co-variance (ANCOVA) was carried out to compare the effects of taping and standard elastic bandage on SEBT. Significance levels were considered at 0.05 for all tests.

\section{Results}

Paired t-test indicated that after taping, reach distances increased significantly in the anterior, anterolateral, lateral and posterior directions whereas after elastic bandage, reach distances increased in the posterior, medial and posteromedial directions (Table1). The results of ANCOVA revealed no difference between the effects of taping and bandage on SEBT measures (Figure2). After applying both taping and standard bandage, traditional COP measures (COP AP\& ML displacements \& velocities) revealed no significant differences except for COP AP velocity which decreased in the taping group (Tables2). Moreover, in both groups, COP-COM moment arm measure did not change considerably after the intervention (Table3).

Table 1. Normalized reach distance measures of SEBT $(\mathrm{cm})$ before and after taping application

\begin{tabular}{lccccc}
\hline $\begin{array}{c}\text { Reach } \\
\text { direction }\end{array}$ & Mean-Pre & Mean-Post & $\begin{array}{c}\text { Mean } \\
\text { differences }\end{array}$ & $\begin{array}{c}\text { Standard } \\
\text { deviation }\end{array}$ & P- value \\
\hline Anterior & 99.95 & 103.82 & 3.876 & 6.018 & $0.017^{*}$ \\
Antero-medial & 99.2 & 101.33 & 2.142 & 5.322 & 0.117 \\
Medial & 89.53 & 93.04 & 3.506 & 7.529 & 0.073 \\
Postero-medial & 84.35 & 87.41 & 3.059 & 6.496 & 0.70 \\
Posterior & 77.25 & 81.54 & 4.282 & 5.677 & $0.007^{*}$ \\
Postero-lateral & 75.91 & 79.05 & 3.142 & 6.882 & 0.078 \\
Lateral & 70.24 & 74.24 & 4.00 & 7.131 & $0.034^{*}$ \\
Antero-lateral & 86.62 & 89.92 & 3.302 & 5.637 & $0.028^{*}$ \\
\hline
\end{tabular}


Table 2. Normalized reach distance measures of SEBT $(\mathrm{cm})$ before and after standard bandage application

\begin{tabular}{lccccc}
\hline \multicolumn{1}{c}{$\begin{array}{c}\text { Reach } \\
\text { direction }\end{array}$} & Mean-Pre & Mean-post & $\begin{array}{c}\text { Mean } \\
\text { differences }\end{array}$ & $\begin{array}{c}\text { Standard } \\
\text { deviation }\end{array}$ & P-value \\
\hline Anterior & 98.74 & 99.82 & 1.088 & 5.524 & 0.429 \\
Antero-medial & 97.94 & 99.06 & 1.123 & 5.630 & 0.423 \\
Medial & 88.25 & 91.99 & 3.735 & 7.072 & $0.045^{*}$ \\
Postero-medial & 81.90 & 86.61 & 4.713 & 6.810 & $0.011^{*}$ \\
Posterior & 76.48 & 81.22 & 4.747 & 5.826 & $0.004^{*}$ \\
Postero-lateral & 75.36 & 78.10 & 2.739 & 7.582 & 0.156 \\
Lateral & 73.08 & 77.09 & 4.018 & 8.169 & 0.060 \\
Antero-lateral & 86.54 & 87.66 & 1.114 & 7.318 & 0.539 \\
\hline
\end{tabular}

Table 3. COP velocity and displacement measures $(\mathrm{mm}, \mathrm{mm} / \mathrm{s})$ before and after McConnell taping application

\begin{tabular}{llllll}
\hline \multicolumn{1}{c}{ COP variables } & Mean-Pre & Mean-Post & $\begin{array}{c}\text { Mean } \\
\text { differences }\end{array}$ & $\begin{array}{r}\text { Standard } \\
\text { deviation }\end{array}$ & P-value \\
\hline AP COP velocity & 73.992 & 63.947 & 10.045 & 18.458 & 0.034 \\
ML COP velocity & 60.004 & 57.584 & 2.420 & 6.118 & 0.122 \\
AP COP displacement & 57.096 & 57.425 & -0.328 & 6.943 & 0.843 \\
ML COP displacement & 44.312 & 42.658 & 1.653 & 7.469 & 0.361 \\
\hline
\end{tabular}

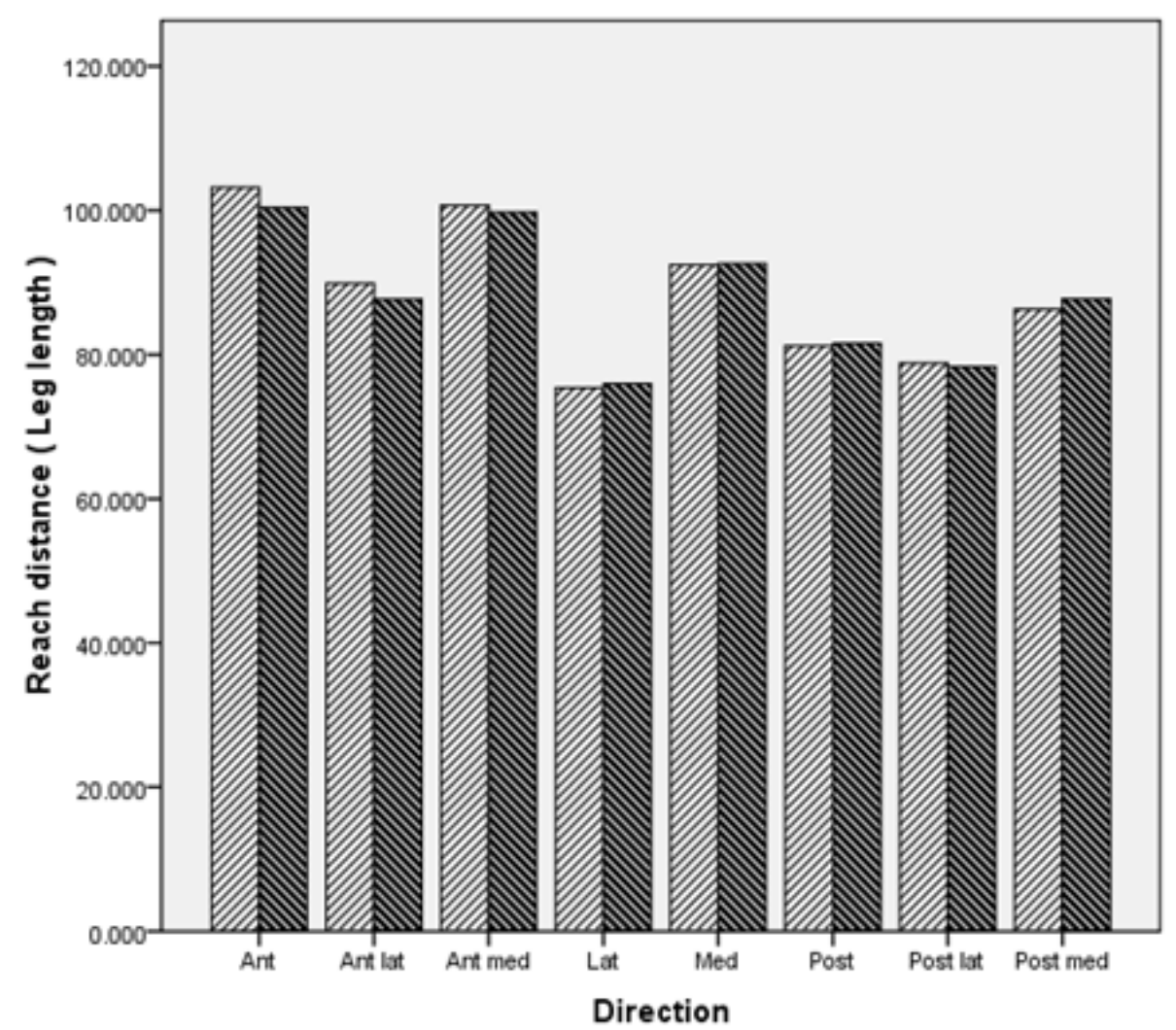

ZTapping

Bandagi

Figure 2. Bar-plot shows no differences between effects of taping and bandage on SEBT. 


\section{Discussion}

Findings of this study revealed that applying McConnell taping and standard elastic bandage improved dynamic postural control but it did not have any effect on static postural control. On the other hand, dynamic measures compared with static variables successfully detected the differences in postural control abilities after the intervention in athletes with PFPS.

Findings of this research indicated that reach distances of SEBT significantly increased in the anterolateral, anterior, lateral and posterior directions due to application of taping, but standard elastic bandage caused an increase in reach distances in the posterior, posteromedial and medial directions. Based on previous research [5], applying McConnell taping and elastic bandage can reduce pain in patients with PFPS; therefore, in the current study the criterion of successful taping and standard bandage was pain reduction on VAS scale. Therefore, increased reach distances after taping might occur due to the following reasons: 1) pain reduction, 2) VMO muscle activity enhancement. As demonstrated in a previous study, healthy participants' anterior direction excursions adopted more vastus medialis activation than other directions [15]. Additionally, some investigations reported that the onset of VMO activity has improved after taping application [16]. This revealed that VMO insufficiency might have caused functional deficits in anterior directions in PFPS patients. Consequently, dynamic postural control improvement especially in anterior and anterolateral reach distances has been due to VMO facilitation. 3) Knee joint stability improvement. It is possible that medial and lateral stabilities are the most sensitive measures of postural control [17, 18]. Therefore, improvement in lateral and medial excursions following taping and standard bandage respectively, can be attributed to magnitude enhancement of VMO activation. Further activation of VMO helps to increase the overall knee joint stability and finally will help the participants to maintain their balance during SEBT.

Previous studies [10, 19] have revealed that patients with anterior cruciate ligament de- ficiency and chronic ankle instability have direction specific deficits when undertaking SEBT. However, the current study showed that reach distances increased in posterior direction after taping and in the posterior and posteromedial directions following standard bandage. Dynamic postural control improvement in these directions might not be related to the effects of two interventions on quadriceps muscle because the greatest quadriceps EMG activity occurred in the anterior direction. These results revealed that improvement in reach distances of SEBT following taping and standard bandage is not necessarily related to direction specific deficits. While posterior reach directions during SEBT need further knee joint flexion, potential improvement in the posterior and posteromedial directions has been due to greater confidence as the result application of taping or standard bandage, as shown in some studies [7].

Findings of this study indicated that there was no significant difference in traditional COP measures (COP AP \& ML displacements, COP ML velocities) following taping and standard bandage except in COP AP velocity that decreased significantly after McConnell taping. Therefore, these results support the hypothesis which explains that the tests performed statically could not contest well the postural control systems in athletes. One systemic review [20] concluded that although CAI patients have a postural control impairment, these alterations have not been detected often with the use of traditional measures. Later studies have assessed the effects of foot supination and pronation on static postural control by Chattex system and dynamic postural control by SEBT [21]. This study showed that balance assessment with static method just detected postural control differences minimally whereas SEBT revealed subtle direction deficits on dynamic postural control. The current study confirms findings of this study because only COP AP velocity measure after taping was changed significantly.

COP is an important method because it represents neuromuscular response to the sway of body's center of mass (COM) but it is not equal to the total body COM [12]. COP-COM moment arm is the distance between COP and 
COM in the transverse plane and considered as an error on postural control mechanisms. This method can detect the subtle differences in static postural control more precisely [22]. Previous research [22] suggests that postural control assessment through time-to-boundary and COP-COM moment arm variable has a more potential to detect postural control impairments compared to traditional COP measure between CAI and healthy controls. However, the results of the current study showed no difference in COP-COM variable following taping and standard bandage. One explanation for the difference between results of this study and those of previous research is considerable differences between the study groups in previous investigations. Also, in this study, subtle differences after the intervention are among reasons for inability of COP-COM variable to identify postural control changes. Additionally, in this study sampling rate of $100 \mathrm{~Hz}$ was used for calculation of COP$\mathrm{COM}$ variable. Probable application of higher sampling rates such as $200 \mathrm{~Hz}$ is more appropriate to detect subtle postural control differences by COP-COM variable.

Statistically, covariance analysis identified no significant differences between the effects of taping and standard bandage on dynamic postural control in athletes with PFPS. However, it seems that taping compared with standard bandage had further influence on dynamic postural control because postural control improvement following taping happened in directions in which patients with patellofemoral pain had neuromuscular deficits (anterior \& anterolateral) or in directions where the most sensitive measures of postural control was established (lateral direction). Findings of this study showed that standard bandage with different mechanisms other than change in the patellar mal-tracking or improvement at VMO activity pattern affects dynamic postural control.

\section{Conclusion}

McConnell taping and standard elastic bandage in athletes with PFPS improve dynamic postural control. Besides, dynamic methods could successfully be used to assess the effects of taping and bandage on postural control. Static variables compared with dynamic measures are not potentially able to detect subtle differences of postural control in athletes with PFPS. In terms of clinical implication, patellar taping and standard elastic bandage application provide useful treatment effects. Because postural control improvement following taping happened in directions with neuromuscular deficits, it was concluded that taping compared with standard bandage had further influence on dynamic postural control. Moreover, dynamic postural control improvement after bandage and taping occurred in different reach distances of SEBT; therefore, it seems that taping and bandage application together are useful in athletes with PFPS for functional performance improvement.

\section{Acknowledgements}

This study was supported by research deputy of Shiraz University of Medical Sciences. We acknowledge Mr. Saeed Heidari and Mrs. Samaneh Neematollahi for their support to collect study data and statistical analysis.

\section{Conflict of Interest}

Authors had no conflict of interest when conducting this research.

\section{References}

1. Myer GD, Ford KR, Barber Foss KD, Goodman A, Ceasar A, Rauh MJ, et al. The incidence and potential pathomechanics of patellofemoral pain in female athletes. Clinical Biomechanics. 2010;25(7):700-7.
2. Pal S, Draper CE, Fredericson M, Gold GE, Delp SL, Beaupre GS, et al. Patellar maltracking correlates with vastus medialis activation delay in patellofemoral pain patients. The American journal of sports 
medicine. 2011;39(3):590-8.

3. Pal S, Besier TF, Draper CE, Fredericson $\mathrm{M}$, Gold GE, Beaupre GS, et al. Patellar tilt correlates with vastus lateralis: vastus medialis activation ratio in maltracking patellofemoral pain patients. Journal of Orthopaedic Research. 2012;30(6):927-33.

4. Herrington L. The effect of patella taping on quadriceps strength and functional performance in normal subjects. Physical Therapy in Sport. 2004;5(1):33-6.

5. Bockrath $\mathrm{K}$, Wooden $\mathrm{C}$, Worrell $\mathrm{T}$, Ingersoll CD, Farr J. Effects of patella taping on patella position and perceived pain. Medicine and science in sports and exercise. 1993;25:989-.

6. Cowan SM, Bennell KL, Hodges PW. Therapeutic patellar taping changes the timing of vasti muscle activation in people with patellofemoral pain syndrome. Clinical Journal of Sport Medicine. 2002;12(6):339-47.

7. Hassan B, Mockett S, Doherty M. Influence of elastic bandage on knee pain, proprioception, and postural sway in subjects with knee osteoarthritis. Annals of the rheumatic diseases. 2002;61(1):24-8.

8. Lephart S, Fu F. The role of proprioception in the treatment of sports injuries. Sports Exercise and Injury. 1995;1(2):96-102.

9. Magee DJ, Zachazewski JE, Quillen WS. Scientific Foundations and Principles of Practice in Musculoskeletal RehabilitationE-Book: Saunders; 2007.

10. Herrington L, Hatcher J, Hatcher A, McNicholas M. A comparison of Star Excursion Balance Test reach distances between ACL deficient patients and asymptomatic controls. The Knee. 2009;16(2):149-52.

11. Aminaka N, Gribble PA. Patellar taping, patellofemoral pain syndrome, lower extremity kinematics, and dynamic postural control. Journal of Athletic training. 2008;43(1):21.

12. Eng J, Winter D. Estimations of the horizontal displacement of the total body centre of mass: considerations during standing activities. Gait \& Posture. 1993;1(3):141-4.
13. Munro AG, Herrington LC. Betweensession reliability of the star excursion balance test. Physical Therapy in Sport. 2010;11(4):128-32.

14. Herrington L. The effect of patellar taping on patellar position measured using ultrasound scanning. The Knee. 2010;17(2):132-4.

15. Aminaka N, Gribble PA. A systematic review of the effects of therapeutic taping on patellofemoral pain syndrome. Journal of Athletic training. 2005;40(4):341.

16. Powers C, Landel R, Sosnick T, Kirby J, Mengel K, Cheney A, et al. The effects of patellar taping on stride characteristics and joint motion in subjects with patellofemoral pain. The Journal of orthopaedic and sports physical therapy. 1997;26(6):286.

17. Friden $T$, Zätterström R, Lindstrand A, Moritz U. A stabilometric technique for evaluation of lower limb instabilities. The American journal of sports medicine. 1989;17(1):118-22.

18. Goldie P, Bach T, Evans O. Force platform measures for evaluating postural control: reliability and validity. Archives of physical medicine and rehabilitation. 1989;70(7):510.

19. Hertel J, Braham RA, Hale SA, OlmstedKramer LC. Simplifying the star excursion balance test: analyses of subjects with and without chronic ankle instability. The Journal of orthopaedic and sports physical therapy. 2006;36(3):131.

20. McKeon PO, Hertel J. Systematic review of postural control and lateral ankle instability, part I: can deficits be detected with instrumented testing. Journal of Athletic training. 2008;43(3):293.

21. Cote KP, Brunet ME, II BMG, Shultz SJ. Effects of pronated and supinated foot postures on static and dynamic postural stability. Journal of Athletic training. 2005;40(1):41.

22. Wikstrom EA, Fournier KA, McKeon PO. Postural control differs between those with and without chronic ankle instability. Gait \& Posture. 2010;32(1):82-6. 